\title{
On ignoring irrelevant dimensions of common familiar stimuli
}

\author{
ILA PARASNIS \\ National Technical Institute for the Deaf, Rochester, New York 14623 \\ and \\ RALPH NORMAN HABER \\ University of Illinois at Chicago Circle, Chicago, Illinois 60680
}

\begin{abstract}
Line drawings of four animals, each differing in size and orientation, were paired with each other in all possible combinations and presented in a successive matching task. In the first experiment, the subjects responded "same" if the stimuli had the same name. The "same" RT was faster for physically identical stimuli than for stimuli that differed on one or two dimensions but still had the same name. "Same" responses were about twice as slow as "different" responses, a finding confirmed in the second experiment, in which subjects responded "same" only to physically identical stimuli. It was suggested that slower "same" responses may result from a general picture-processing strategy in which differences were noticed faster than similarities.
\end{abstract}

Substantial evidence now exists to show that pictures or line drawings of objects, shapes, or scenes are coded in short-term memory differently from verbal stimuli (Paivio, 1975; Posner \& Mitchell, 1967; Shepard, 1975). To study the nature of visual coding processes in shortterm memory, Klatzky and Stoy (1974) measured reaction time (RT) in a matching task by briefly presenting line drawings of common familiar objects successively at interstimulus intervals (ISIs) of $.3,2$, or $4 \mathrm{sec}$. The subjects were told to respond "same" if both drawings were physically identical, if one was the mirror image of the other, or if both had the same name but different visual structures. One intriguing finding of this study was that average RTs for identical and mirror image matches did not differ from each other, regardless of the ISIs including the short ISI of $300 \mathrm{msec}$. Klatzky and Stoy explained their result by postulating that familiarity can influence a specialized "orientation-independent comparison process" within short-term memory, which allowed identity and mirror matches to be carried out with equal speed. Since Klatzky and Stoy found no difference in average RT for identity and mirror matches at an ISI of $300 \mathrm{msec}$, it seems that familiarity can

This research was conducted when both authors were affiliated with the University of Rochester. We thank Dennis Fisher for his kind permission to use the tachistoscope at the Aberdeen Proving Grounds and, when it was on loan at the University of Rochester, Alfred O. Dick for his substantial help in programming and data analysis, and James Clark for drawing superb stimuli. Requests for reprints should be sent to: Ila Parasnis, Department of Communication Research, National Technical Institute for the Deaf, One Lomb Memorial Drive, Rochester, New York 14623. influence processing of information at an early stage in short-term memory.

We explored this possibility in an RT study using line drawings of common animals that varied on two familiar dimensions, size and orientation. If specialized processing of common familiar objects occurs in short-term memory, the "same" RT for identical matches should not differ from that for mirror image matches or for large-small size matches. Moreover, it is possible that the familiarity with seeing animals in different orientations at different distances can influence the specialized processing in such a way that both dimensions can be ignored in matching animals. Thus, average RT for identical matches may not differ from that for matches in which animals differ in size as well as orientation. On the other hand, if common familiar objects are initially coded in short-term memory in the same manner as other nameable multidimensional stimuli are, increasing the number of stimulus dimensions should result in an increment in RT (see Nickerson, 1972). Furthermore, even if dimensions are made irrelevant for the naming task, there should be an increment in RT compared with that for identical matches, since research shows that irrelevant stimulus dimensions cannot be completely ignored (e.g., Egeth, 1966).

\section{EXPERIMENT 1}

\section{Method}

Subjects. Six enlisted personnel at the Aberdeen Proving Grounds, Maryland, participated in the study. All had normal vision.

Stimuli and Apparatus. Line drawings of four different animals varying in size (large or small) and in orientation (facing left or right) were prepared (see Figure 1). Only the outlines 

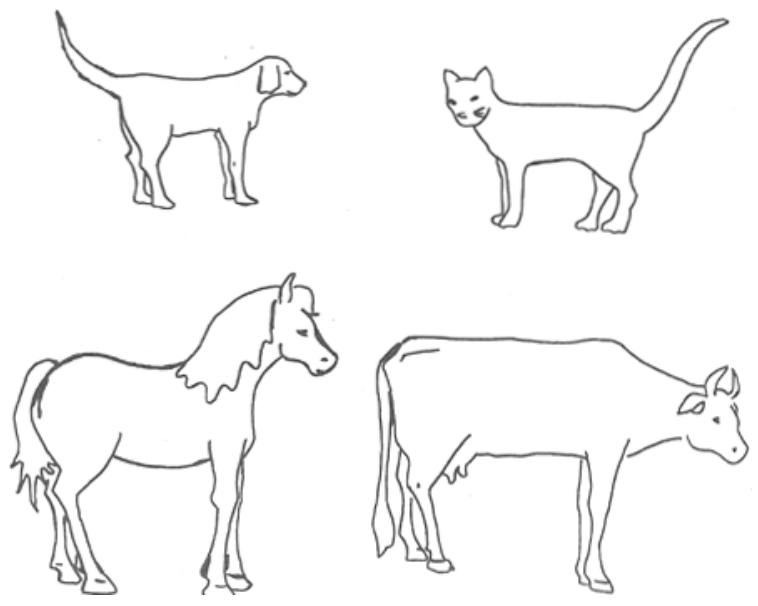

Figure 1. Examples of line drawings of four animals used in this study, illustrating differences in small and large size and differences in orientation.

were used, so that no orientation or size invariant information was present within the stimuli that might confound the results in comparing identity matches with other matches. The 16 different line drawings $(4 \times 2 \times 2)$ were paired with each other in all possible combinations, yielding 16 identical and 120 different pairs of stimuli. A three-channel Scientific Prototype Automatic tachistoscope was used to present the slides of the stimuli. Luminance was set at $20 \mathrm{~cd} / \mathrm{m}^{2}$ for all channels. Two channels were used to expose the stimuli, and the third channel contained a central fixation cross that was on during the intertrial intervals and the ISIs. In each trial, a stimulus was presented for $100 \mathrm{msec}$ followed by one of three ISIs, which was followed by the second stimulus for $100 \mathrm{msec}$. Subjects responded to the stimuli by pressing one of the two keys under their index fingers. A HewlettPackard electronic counter was started with the onset of the second stimulus and was stopped by the subject's response. RT was measured to the nearest $1 \mathrm{msec}$ and printed out by a Hewlett-Packard digital recorder.

Experimental design and Procedure. The subjects were instructed to press the "same" key if the pair of the stimuli were of the same animal and to ignore size and orientation differences. They were told to press the "different" key when the second stimulus was of a different animal.

In each testing session, a total of 240 trials were given. The trials were structured in such a way that $50 \%$ of the time the subject should press the "same" key and 50\%, the "different" one. There were 40 possible combinations of stimuli that had the same animal name and thus would lead to a "same" response. These consisted of 16 physically identical pairs, eight sameanimal pairs that differed in size, eight same-animal pairs that differed in orientation, and eight same-animal pairs that differed in both size and orientation. All stimuli were repeated three times, to produce 120 "same" trials in the session. There were 96 possible different-animal pairs, which were each presented once. Twelve pairs from those were randomly selected and repeated twice to produce 120 "different" trials. The subjects were tested in four different sessions on 4 different days. The 1 st day was a practice session for all subjects, in which the stimuli were presented with an ISI of 500 msec. On the subsequent 3 days, the subjects were tested with ISIs of $250 \mathrm{msec}, 500 \mathrm{msec}$, and $750 \mathrm{msec}$. The order in which ISIs were presented was counterbalanced over days and over subjects. Within each session, the 240 trials were given in three blocks with a 2 -min rest period between each block. The order of blocks was counterbalanced across subjects. Half of the subjects used the index finger of their preferred hand to press the "same" key and half to press the "different" key. The "same" and "different" trials were randomly assigned, with the limitation that stimuli requiring one type of response not be repeated more than three times. However, twice at random, they were repeated four times in a row in order to prevent subjects' guessing.

\section{Results and Discussion}

The RTs from the practice sessions were excluded from final analyses, and only the correct responses from test sessions were used. The average error rate of subjects was $2.2 \%$. The main results of the analysis of variance of the data are plotted in Figure 2. Since no significant differences in RTs given at the three different ISIs were found, nor any significant interactions with ISIs for any of the comparisons reported below $(p>.18)$, the data in Figure 2 are collapsed over ISIs. The "same" and "different" responses were assigned to categories according to the number of dimensions on which the pairs matched or mismatched. The number of observations per subject at each data point varied from 72 to 144 trials, depending upon the type of response and stimulus category.

A trend analysis of the data for the "same" responses showed that the mean RT for physical matches (AOS) was significantly faster than that for matches in which one dimension differed [i.e., mirror image matches (AO), and large-small size matches (AS); $F(1,5)=15.80$, $\mathrm{p}<.01]$ and that for matches in which two dimensions were different and only the animal name was the same $[F(1,5)=8.99, p<.03]$. The mean $R T$ for matches in which one dimension was different was faster than that for matches in which two dimensions were different, but the difference in mean RTs did not reach significance $[F(1,5)=5.44, p<.07]$.

A trend analysis of the data for "different" responses showed that when pairs of animals differed on every dimension (AOS), the mean RT was faster than when only the animal name was different (A) $[F(1,5)=8.41$, $\mathrm{p}<.03]$. When pairs of animals differed in animal name and orientation (AO) or in animal name and size (AS), the mean RT was also significantly faster than when

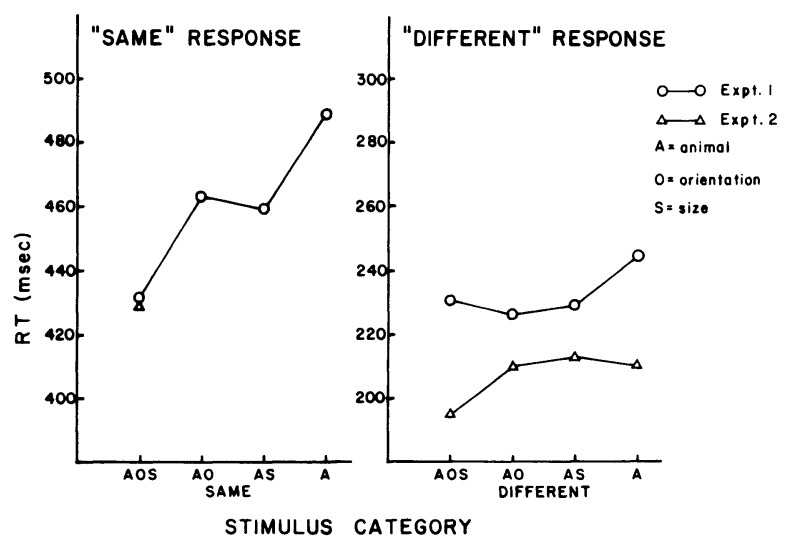

Figure 2. Mean RTs for "same" and "different" responses in Experiments 1 and 2. Stimulus categories indicate the dimensions on which the pairs were the same or different. 
only the animal name was different $(\mathrm{A})[\mathrm{F}(1,5)=13.5$, $\mathrm{p}<.01]$. There was, however, no significant difference between the average RTs for pairs that differed on all dimensions and those that differed on two dimensions $[\mathrm{F}(1,5)=.13, \mathrm{n} . \mathrm{s}$.$] .$

The results of this study supported the earlier findings that irrelevant dimensions cannot be completely ignored. No orientation-independent or size-independent comparison processes as suggested by the Klatzky and Stoy (1974) hypothesis seemed to have been employed, since the identical matches were faster than the other "same" matches. One unusual finding of this study was that the "same" responses were slower than the "different" responses. The fastest "same" response when all dimensions were identical took twice as long as the fastest "different" response when stimuli differed on all dimensions. This result seems to be quite contrary to the general findings of RT studies (Nickerson, 1972). It should be noted that the result was quite robust, since it was obtained in spite of an experimental condition adverse to it. We had repeated the 40 "same" stimuli three times, whereas most of the "different" pairs (96) were presented only once. Repetition of the "same" pairs should have made the "same" RT faster than usual (Williams, 1972); however, the "same" RT was still quite slow.

One explanation may be that the instructions or the task demands made subjects adopt a cautious strategy in responding "same," which resulted in an overall slowing down of the "same" response. The following experiment tested this possibility by simplifying the task for the "same" response. Subjects were told to press the "same" key only if stimuli were physically identical.

\section{EXPERIMENT 2}

\section{Method}

Subjects. Six graduate students at the University of Rochester, Rochester, New York, participated in the study. All had normal vision.

Stimuli and Apparatus. The same stimuli and apparatus were used as in Experiment 1.

Experimental design and Procedure. The 16 identical pairs were each repeated seven times, and eight randomly selected pairs were repeated one more time, so that the number of "same" trials reached 120 . One hundred and twenty different pairs were each presented once, to produce a total of 240 trials. The subjects were instructed to press the "same" key if the pair of stimuli were identical and the "different" key if the second stimulus differed from the first one in size, orientation, or animal name. The procedure of testing subjects was the same as that in Experiment 1.

\section{Results and Discussion}

The RTs from the practice sessions were excluded from the final analyses, and only the correct responses from test sessions were analyzed. The average error rate was $3.13 \%$. The main results of the analysis of variance of the data are plotted in Figure 2. Since no significant differences in RTs given at the three ISIs were found, nor were any significant interactions of ISIs with any of the comparisons reported below ( $p>.27$ ), the data in Figure 2 are collapsed over ISIs. The "different" responses were assigned to categories as before, according to the number of dimensions on which the pairs mismatched. The number of observations per subject at each data point varied from 72 to 360 trials, depending upon the stimulus category and the type of response.

The data analysis showed that the average RT for the "same" response to identical pairs was $431 \mathrm{msec}$, very similar to the "same" response $(429 \mathrm{msec})$ to identical matches in Experiment 1.

A trend analysis of the data for the "different" responses showed results similar to those found in Experiment 1. The mean RT was faster when all three dimensions differed (AOS) than when pairs differed on two dimensions, namely, animal name and orientation (AO), animal name and size (AS) $[F(1,5)=75.51$, $\mathrm{p}<.001]$, or when only the animal name differed (A) $[F(1,5)=79.69, p<.001]$. There was no significant difference between the categories in which only the animal name was different or in which the animal name and size or animal name and orientation were different $[\mathrm{F}(1,5)=.45, \mathrm{n} . \mathrm{s}$.$] .$

These results confirmed the findings from Experiment 1 . No differences due to ISIs were found, and the average RT for the "same" response for identical matches in both experiments differed by only 2 msec. The average RT for the "different" response was faster when all dimensions differed than when only one dimension was different. Furthermore, the average RT for the "same" response was still about twice as slow as that for the "different" response. By repeating only 16 identical stimuli to make 120 trials as opposed to 40 in Experiment 1, we were again allowing for a repetition effect to occur, and, again, in spite of it, the mean "same" RT was slower than the mean "different" RT. Since we asked the subjects in this experiment to respond "same" only to identical matches and still found a slower mean "same" RT, the possibility that the instructions in Experiment 1 affected subjects' strategies is remote.

\section{GENERAL DISCUSSION}

The results of this study supported the hypothesis that information from irrelevant dimensions of familiar objects cannot be ignored in the early stages of information processing and that its interference results in an increase in the "same" RT in a matching task. Further, as the number of irrelevant dimensions on which the stimulus pair was different increased, the "same" response became slower. Conversely, as the number of irrelevant dimensions on which the stimulus pair was the same increased, the "different" response became slower. These results are contrary to Klatzky and Stoy's (1974) findings. A specialized comparison process influenced by familiarity did not seem to operate when a matching task was done in less than $1 \mathrm{sec}$. Our ISI values bracketed the shortest one in the Klatzky and Stoy study, but all were shorter than their two longer ISI values. Therefore, the present results do not rule out the possibility that a specialized comparison process influenced by familiarity may be activated in short-term memory at longer ISIs. 
It should be noted here that no significant effect of ISI on RTs to "same" or "different" stimuli was found in the present experiments, which is contrary to the general findings reported in the literature. Since this "nonsignificant" effect of ISI as well as other significant effects found in the first experiment were replicated in the second experiment employing different subjects, it seems likely that our failure to find the ISI effect is related to the nature of stimuli and the task used in the present research. The stimuli were simple line drawings of only four highly familiar animals, and the task in both experiments did not require naming them. It is possible that under those conditions, visual coding of the pictures can occur within $250 \mathrm{msec}$ and be maintained for less than $1 \mathrm{sec}$, so that the comparison process can be carried out efficiently regardless of the ISI. Whether this result can be generalized to experiments in which there is an increase in the complexity of pictures, category width, or the number of different categories represented by pictures needs to be investigated.

In both experiments, it was found that the average "same" response was about twice as long as the average "different" response. This result is unusual, since RT studies using multidimensional stimuli have generally reported slower "different" responses (Nickerson, 1972). Perhaps this discrepancy can also be resolved by noting that the multidimensional stimuli in our experiment were pictorial representations of highly familiar, common objects. Recently, Tversky (1979) found that while comparing a pair of simultaneously presented pictures that were two different drawings of the same object, subjects gave "same" responses slower than "different" responses. She suggested that this reversal in results might be due to the subjects' doublechecking to determine if the stimuli referred to the same object. This explanation may be applied to the results of the first experiment; however, it cannot account for the results of the second experiment. In Experiment 2, the subjects responded "same" only to identical pairs, for which such double-checking would not be necessary, and still gave slower "same" responses than "different" responses. Thus Tversky's hypothesis cannot adequately explain the results of both experiments.

It is possible to explain our results by referring to the hypothesis suggested by some studies of picture processing that have used line drawings of scenes (Attneave, 1957; Biederman, 1972; Loftus \& Mackworth, 1978). This hypothesis postulates that subjects may build a visual schema of the whole scene and information that is unusual or different is processed faster than that which fits in with the general schema. If this strategy is a general picture-processing strategy, it may occur in processing a single picture or in matching two pictures. Thus, we can speculate that in our experiments subjects created a schema of the whole stimulus and compared it with the subsequent picture. They processed differences faster than similarities, as the pictureprocessing studies suggest, and thus "different" responses, in general, were faster than "same" responses. Such a hypothesis is also consistent with our finding that when the stimuli were identical or maximally different, they were processed the fastest.

\section{REFERENCES}

Atrnenve, F. Transfer of experience with a class-schema to identification learning of patterns and shapes. Journal of Experimental Psychology, 1957, 54, 81-88.

Biederman, I. Perceiving real world scenes. Science, 1972, 177, 77-80.

Egeth, H. Parallel versus serial processes in multidimensional stimulus discrimination. Perception \& Psychophysics, 1966, 1, 245-252.

Klatzky, R., \& Stoy, A. Using visual codes for comparison of pictures. Memory \& Cognition, 1974, 2, 727-736.

Loftus, G., \& Mackworth, N. Cognitive determinants of fixation location during picture viewing. Journal of Experimental Psychology: Human Perception and Performance, 1978, 4, 565572.

Nickerson, R. Binary classification reaction time: A review of some studies of human information-processing capabilities. Psychonomic Monograph Supplements, 1972, 4, 275-317.

Palvio, A. Coding distinctions and repetition effects in memory. In G. H. Bower (Ed.), The psychology of learning and motivation (Vol. 9). New York: Academic Press, 1975.

Posner, M., \& Mitchell, R. Chronometric analysis of classification. Psychological Review, 1967, 74, 392-409.

ShePARD, R. Form, formation and transformation of internal representations. In $\mathbf{R}$. Solso (Ed.), Information processing and cognition: The Loyola symposium. Hillsdale, N.J: Erlbaum, 1975.

Tversky, B. Pictorial representations in adults and children. Quarterly Journal of Experimental Psychology, 1979, 31, 397 408.

Williams, J. The effect of practice with controlled stimulus pairs on same-different judgments. Journal of Experimental Psychology, 1972, 96, 73-77.

(Received for publication June 9, 1981.) 